\title{
An Efficient Virtual Trachea Deformation Model
}

\author{
Cui Tong ${ }^{a}$ and Zhao Haifeng \\ Nanjing Research Institute of Electronics Engineering, 210007, Nanjing, China \\ Science and Technology on Information Systems Engineering Laboratory, 210007, Nanjing, China
}

\begin{abstract}
In this paper, we present a virtual tactile model with the physically based skeleton to simulate force and deformation between a rigid tool and the soft organ. When the virtual trachea is handled, a skeleton model suitable for interactive environments is established, which consists of ligament layers, cartilage rings and muscular bars. In this skeleton, the contact force goes through the ligament layer, and produces the load effects of the joints, which are connecting the ligament layer and cartilage rings. Due to the nonlinear shape deformation inside the local neighbourhood of a contact region, the RBF method is applied to modify the result of linear global shape deformation by adding the nonlinear effect inside. Users are able to handle the virtual trachea, and the results from the examples with the mechanical properties of the human trachea are given to demonstrate the effectiveness of the approach.
\end{abstract}

\section{Introduction}

Trachea surgery is still one of the most demanding fields in surgery to cure tumours, that is, one of the leading causes of death worldwide. Teaching and learning trachea surgery and internal trachea anatomy have been restricted to merely watching trachea operations and videos, studying conventional diagnostic images [1].

The most challenging task in virtual trachea surgery is to build an efficient and real-time force/deformation model in the surgery simulation. However, high-quality feedback demands a high level of physical realism as well as a high update rate in rendering, which are often conflicting requirements. Over the past two decades, many models have been developed to simulate deformable objects for a broad range of computer graphics applications. An alternative approach to physically based deformation modelling are spring-based methods, which model a deformable object as a collection of mesh nodes connected by springs in a mesh skeleton. In general the computations involved in spring models are relatively simple and easy to implement. But the human trachea is non-isotropic and made up of anisotropic materials, so that it is difficult to render physically believable behaviours. In addition, the large number of springs and mass points composing the model makes it fastidious to define parameters and control the elongation, flexion and torsion at a macroscopic level [2].

The proposed approach is a physically based model more apt for modelling force and deformation under simple contact or multiple contact conditions. The paper presents a skeleton model to simulate the trachea deformation in virtual surgery and we discuss implementation and experimental results including the single finger contact.

\footnotetext{
${ }^{\mathrm{a}}$ Corresponding author: cuitong_seu@sina.cn
}

\section{Trachea skeleton model}

Due to the complex shape and non-homogeneous material properties, we give some assumptions about human trachea model and restrict our study in this paper to the trachea model scenarios. And a novel skeleton modelling approach is introduced to simulate the trachea deformation under the external force.

\subsection{Assumptions about trachea skeleton}

In this paper, we assume human trachea is elastic model and mainly made of two homogeneous materials cartilage rings and muscular tissues. And each material has a constant elastic coefficient, in which the external pressure distributes evenly.

In the real trachea, two or more of the cartilages often unite, partially or completely, and they are sometimes bifurcated at their extremities. And the outer surfaces of the cartilage rings are flattened in a vertical direction, but the internal are convex, the cartilages being thicker in the middle than at the margins [3] as shown Fig.1. However, to simplify the case, the cartilage rings, which have the same geometry shape and material properties, are placed horizontally above each other and separated symmetrically by narrow intervals.

As the cartilage material is stiffer than the muscular tissue to resist the external forces, we only consider the effect of the cartilage rings under the press and ignore the elasticity of the muscular tissue between the cartilage rings. In addition, the gravity force of the trachea itself as a thin shell tube is much smaller than the react forces, and therefore the gravity force is neglected in the experiments. And the larynx connecting with the trachea is stiff solid, 
when it is pulled by the stretch from the trachea, its shape keeps invariable but its displacement is changed. Similarly, the displacement of the primary bronchi with the trachea fork end is proportional to the stretch from the trachea end.

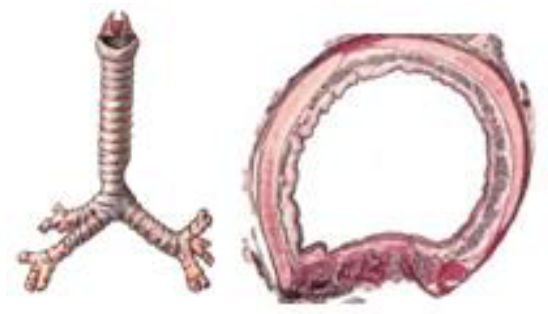

Figure 1. Trachea and its crossing-section with cartilage ring

Duffing Equation [4] is used to characterize the nonlinear force response $\mathrm{F}$ and the magnitude $\mathrm{D}$ of the displacement vector of contact point at a quasi-static state, which is the qualitative analysis of the deformation behaviour manually during the operation.

$$
\|F\|=m_{0}\left|\omega_{0}^{2} D+0.75 \beta_{0}^{2} \varepsilon D^{3}\right|
$$

where $m_{0}$ is the mass of the virtual rigid tool, and $\omega_{0}, \beta_{0}^{2} \varepsilon$ are the parameters of linear and nonlinear restoring terms in the Duffing Equation respectively.

\subsection{Trachea skeleton model}

The skeletal trachea is a bony tube that commences at the larynx and bifurcates into the primary bronchi, and is an important part of the vertebrate respiratory system. It is flexible and forms the vacuum trunk of an upside-down tree. The trachea is mainly made up of the elastic smooth ligament tissues with about 15-20 incomplete C-shaped cartilaginous rings, which keeps the trachea open so the head and neck may twist and bend during the process of breathing. The each cartilage of the trachea occupies the anterior two-thirds, being deficient behind, where the tube is completed by unstrapped muscular fibbers. The trachea has an inner diameter of 20-25 mm and a length of 10-16 cm in adults [5]. Seen in Fig. 2.
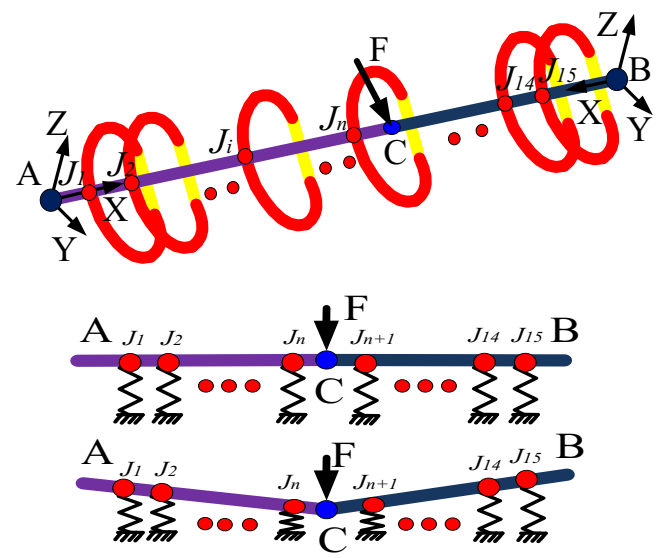

Figure 2. The trachea skeleton

What is meant by skeleton decisions and skeleton design is the process of arriving at a suitable system to support a form or shape and to prevent it from collapsing
[6]. In order to compute the real-time global deformation of the trachea efficiently, we present a unique skeleton model for the trachea to simulate force and deformation when the contact occurs. The trachea skeleton involves a serial of highly elastic cartilage rings (they may become calcified in advanced life), the ligament layer between the cartilage rings, and the smooth muscle connecting the two ends off the incomplete cartilaginous ring open in the back.

And the ligaments tend to be more flexible and be able to accommodate movement, while cartilage rings tend to be rigid and unable to accommodate movement to avoid some damage to the skeleton. So the skeleton can guarantee the stability by being placed in such a way as to procure balance when contacted.

As the ligament tissue is a longitudinal and transverse layer, once a contact point $\mathrm{C}$ is formed, between the trachea fork end $\mathrm{A}$ and the larynx end $\mathrm{B}$, two linear continuous ligament beams $\mathrm{AC}$ and $\mathrm{BC}$ are established at the same time, which are parallel with the central line of the tube and joint the cartilage rings through the ligament layer. And the bottom elastic muscular layer resists the pressure and supports the cartilage rings, which can be viewed as a spring mat to sustain a serial of two-hinge arches. To simplify this case, we design a continuous ligament beam with spring supports to simulate the skeleton deformation as shown in Fig. 2.

In our application, the force $F$ applied to the contact point can be regarded as applied to the common end $\mathrm{C}$ of two separate beams. Establish two coordinate systems A (B)- $x y z$ for the two beams respectively, where the origin is set at one fixed end A (B) of the beams, and $\mathrm{x}$-axis is along the central line of each beam before it is bent and pointing to the contact point $\mathrm{C}$. The $\mathrm{y}$-axis follows the bending force direction, and the $z$ - axis is orthogonal to both $x$ and $y$ axis following the right-hand rule.

The contact force $\mathrm{F}$ as the sum of the force FAC and FBC applied to the beam $\mathrm{AB}$ and $\mathrm{BC}$, so the deformation of each beam can then be computed separately. In this case, the two forces can be both decomposed into the bending case along $x$-axis direction and compression/expansion case along $y$-axis direction. According to the Bernoulli-Euler bending beam theory, the relation between the distributed forces of each beam can be expressed as:

$$
\begin{gathered}
F_{A C}^{x}: F_{B C}^{x}=\frac{D_{x} S_{B}}{L_{A C}}:-\frac{D_{x} S_{B}}{L_{B C}}=-\frac{L_{B C}}{L_{A C}} \\
F_{A C}^{y}: F_{B C}^{y}=\frac{3 D_{y} E_{B} I_{B}}{L_{A C}{ }^{3}}: \frac{3 D_{y} E_{B} I_{B}}{L_{B C}{ }^{3}}=\frac{L_{B C}{ }^{3}}{L_{A C}{ }^{3}}
\end{gathered}
$$

where $D_{x}, D_{y}$ are the displacements of the contact point $\mathrm{C}$ along $x$-axis and $y$-axis; LAC, LBC are the lengths of two beams, EB and IB are the Young module and the moment of inertial of the ligament beam, and SB is the crosssection area, whose diameter is the thickness of the ligament layer. Note that plus in the equation (2) show one beam is contracted and the other is extended.

The two ligament beams connect the cartilage ring $i$ $(\mathrm{i}=1,2, \ldots, 15)$ with the joint $J_{i}$. So the pressure $P_{i}$ from the ligament beams can not only make the joint $J_{i}$ 
displacement but also change the shapes and positions of its cartilage ring. For each cartilage ring open in the back, its two ends are connected by a muscular bar viewed as a spring tie, which allows the cartilage ring to stretch and contract in breathing or swallowing. Seen in Fig. 3. We can compute general contact/deformation effects resulted from the beams or arches based on Structure Analysis Theory, as detailed in the following sections.

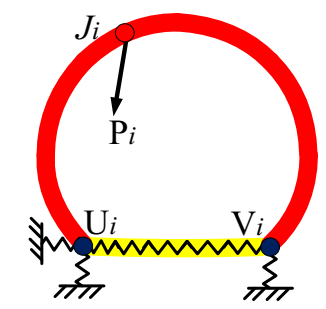

Figure 3. The two- hinge cartilage arch model

\subsection{Skeleton shape deformation}

We model the shape deformation of the elastic trachea under the contact force from the virtual rigid object. As the trachea skeleton is a statically indeterminate structure with redundant, Force Method as a general approach is used to analyze the skeleton with the determination of the degree of static indeterminacy and identification of the redundant [7].

In this section, we consider how to use Force Method to compute the deformation of the joint supports under the continuous ligament beam, which is commonly subjected to transverse loads. We shall ensure the degree $n$ of statically indeterminate beam, so it is necessary to find $\mathrm{n}$ stress resultants in the beam from conditions of compatibility of displacements. That is, the beam is statically determinate when $n$ external reaction supports have to be released. In this case, each redundant $i$ in the continuous ligament beam is assumed to be replaced by the joint force $P_{i}$, so a stable and statically determinate skeletons becomes a simply supported beam with the fixed end as shown in Fig. 4.

The $n$ support forces will be introduced at released supports from $J_{i}$ to $J_{n}$ along the continuous beam; thereby the beam comprises $n$ spans of length 1 and one span of length a near the contact point. And at any joint $J_{i}$ $(1 \leq i \leq n)$ of the continuous beam there is a joint force $P_{i}$, which produces the joint deflection in the beam. The joint bending moment $m_{i}$ from the support $i$ and the load bending moment $m_{F_{A C}}^{y}$ can be calculated by the compatibility equations [8] based on Diagrammatic Multiplication Method [7]. Note the contact force component $F_{A C}^{x}$ have no moment effect on the continuous beam.

$$
\begin{gathered}
m_{i}=i l p_{i} \\
m_{F_{A C}^{y}}=(n l+a) F_{A C}^{y}
\end{gathered}
$$

And each flexibility influence coefficient means a displacement made by a unit support force, which is along the direction of the support force on one joint.

$$
\delta_{i j}=\delta_{j i}=\int \frac{m_{i} m_{j}}{E_{B} I_{B}} d x=\frac{(3 j-i) i^{2} l^{3}}{E_{B} I_{B}} \quad(1 \leq i \leq j \leq n)
$$

where the first subscript of this influence coefficient means the index of the joint site and its displacement vector is along the direction of the elastic force of the joint spring support, and the second subscript denotes the index of the support force. The continuous beam has a constant Young's Module EB, moment of inertia IB.

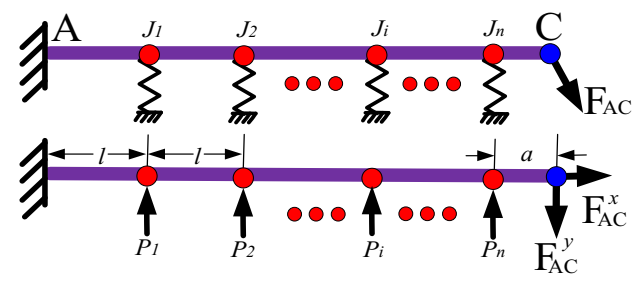

Figure 4. Continuous beam model and redundant force

And then we set up the flexibility matrix $\delta$ of the released beam to express $\mathrm{n}$ simultaneous equations as following.

$$
\delta=\left[\begin{array}{cccc}
\delta_{11}+\frac{1}{k} & \delta_{12} & \cdots & \delta_{1 n} \\
\delta_{21} & \delta_{22}+\frac{1}{k} & \cdots & \delta_{2 n} \\
\delta_{n 1} & \delta_{n 2} & \cdots & \delta_{n n}+\frac{1}{k}
\end{array}\right]
$$

Similarly, the deflection $\Delta_{i F_{A C}^{y}}$ at the release $i$ caused by the applied contact force $F_{A C}^{y}$ are calculated as

$$
\Delta_{i F_{A C}^{y}}=\int \frac{m_{i} m_{F_{A C}}^{y}}{E_{B} I_{B}} d x=\frac{3(n+a)-i}{6(n+a) E_{B} I_{B}} i^{3} l^{3} F_{A C}^{y}
$$

where $m_{F_{A C}}^{y}$ is the bending moment distribution in the released skeleton due to the applied loading.

The compatibility function under the deformation condition at the releases is the following equation, and the joint force $P_{i}$ at each resultant $i$ is what we want to find.

$$
\sum_{i=1}^{n} \delta_{i j} P_{i}+\Delta_{i F_{A C}^{y}}=0
$$

That is,

$$
\left[\begin{array}{cccc}
\delta_{11}+\frac{1}{k} & \delta_{12} & \cdots & \delta_{1 n} \\
\delta_{21} & \delta_{22}+\frac{1}{k} & \cdots & \delta_{2 n} \\
\delta_{n 1} & \delta_{n 2} & \cdots & \delta_{n n}+\frac{1}{k}
\end{array}\right]\left[\begin{array}{c}
P_{1} \\
P_{2} \\
\cdots \\
P_{n}
\end{array}\right]+\left[\begin{array}{c}
\Delta_{1 F_{A C}^{y}} \\
\Delta_{2 F_{A C}^{y}} \\
\cdots \\
\cdots \\
\Delta_{n F_{A C}^{y}}
\end{array}\right]=\left[\begin{array}{c}
0 \\
0 \\
\cdots \\
\cdots \\
0
\end{array}\right]
$$

So we can obtain the displacement $d_{i}^{y}$ of the each joint $J_{i}$, which is always along the direction of the joint load component $F_{A C}^{y}$.

$$
d_{i}^{y}=\frac{P_{i}}{k}
$$


where the internal stiffness $k$ is achieved by the combination of the cartilage materials and the bottom muscular layer. To simplify the case, we let $k$ equal to the elastic coefficient $k_{M}$ of the muscular layer approximately because the cartilage material is more rigid than the muscular tissue.

However, the external force FAC applied to a ligament beam is not necessarily normal to the beam central line.

\subsection{Global deformation rendering}

The trachea model has a general surface and let $S$ be the set of its surface. Such a surface $S$ can be partitioned into many single-surface patches $S_{k}$, which represents the region between two neighbourhood cartilage rings. As shown in Fig.5, the contact point (if it exists in this patch), two joints $\left(J_{i}, J_{i}+1\right)$ and four ring ends $\left(V_{i}, V_{i}+1, U_{i}, U_{i}\right.$ $+1)$ are viewed as the key points in this patch $S_{k}$. So we can divide the patch $S_{k}$ into three regions $G_{i}\left(U_{i}, U_{i}+1, J_{i}\right.$, $\left.J_{i}+1\right), G_{i}+1\left(J_{i}+1, J_{i}, V_{i}, V_{i}+1\right)$ and $G_{i}+2\left(V_{i}, V_{i}+1, U_{i}\right.$ $\left.+1, U_{i}\right)$ according to the distribution of the ligament beam and the muscular layer.

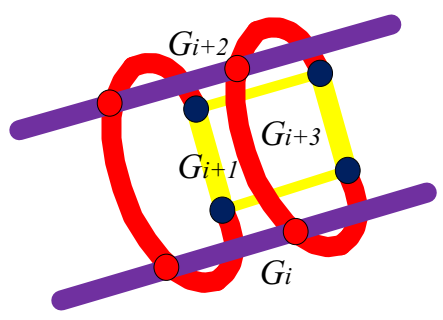

Figure 5. The Region distributions in the $S_{k}$ patch

Phong shading method is an interpolation method, which is used for linear interpolation of vectors at vertices bounding a polygon across internal points of the polygon [9]. In each region $G$ of the surface patch $S_{k}$, let $\theta_{i}$ represents the displacement angle of key point $i$, which means the angle between the direction of displacement and the outward normal direction of the key point $i$. So the directions of displacement vectors across the region $G$ are obtained by linearly interpolating the displacement angles $\theta_{i}$ in the same region, and the magnitudes of displacement vectors across the region $G$ are obtained by linearly interpolating the magnitudes of displacement vectors of the key points in this region. We achieve the deformed shape by performing the above shading on all surface patches. The global shape deformation of the trachea model obtained from interpolating these displacements is smooth and satisfies that the closer a surface point to the contact point, the greater the deformation change is at this point [4].

\section{Implementation and test results}

In this section, we show a virtual trachea surgery method to simulate the actual surgical cases. Our implementation is done in a virtual environment on a PC with dual Intel Xeon $3.0 \mathrm{GHz}$ Processors and $2 \mathrm{~GB}$ system RAM. The ultimate goal of this study is to obtain a real-time virtually simulated result of the trachea deformation. In order to accomplish this, we apply the virtual fingers and tools controlled by keys on the computer keyboard or via a haptic device for the realtime interaction to test the simulation algorithm.

The operator controls the virtual rigid finger move and touch the model surface. There, we assume the finger can press or draw the trachea and move with it together. And the Fig. 6 shows the contact force/deformation rendering between the rigid finger and the trachea. As for the compliant motion, we will research in the future work.

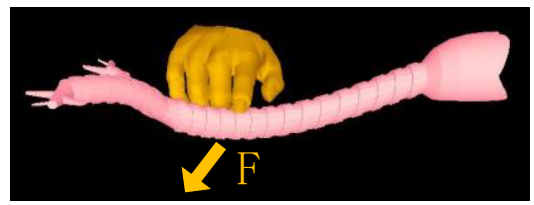

(a) The haptic rendering under the normal force

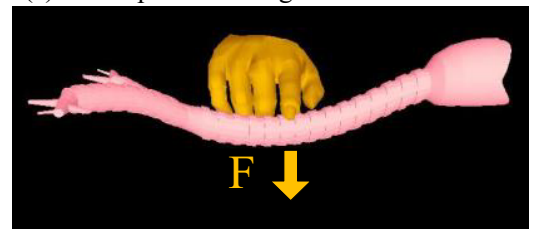

(b) The haptic rendering under the force pointing to left-down direction

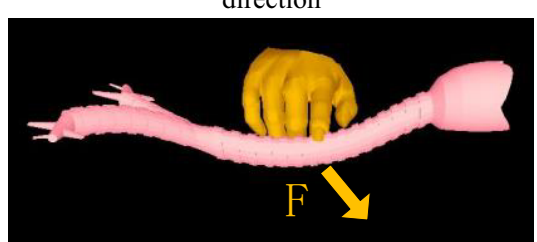

(c) The haptic rendering under the force pointing to right-down direction

Figure 6. Trachea haptic rendering in the simple contact

Fig. 7 describes the above experiment and represents the relations between the force and deformation in the experiments. When the contact occurs, each joint load $P_{i}$ (from the 1 th to the 15th) has two components: $P_{i}^{x}$ and $P_{i}^{y}$. Here during the pressing, the value of each load $P_{i}$ is changed from zero to the maximum. The nearer to the contact point, the larger the magnitude of the force component $P_{i}^{y}$ is. Here, the 6th joint achieves the largest force $P_{6}^{y}$ (in the Fig. 7a). And then the other force component $P_{i}^{x}$ has the approximately same value at each joint. Similar to the case of the joint load $\mathrm{Pi}$, the deformation $D_{i}\left(D_{i}^{x}, D_{i}^{y}\right)$ of each joint $i$ can also be shown in Fig. 7b.

Here the deformation component $d_{i}^{x}$ of each joint is proportional to the distance from the contact point. Due to the normal force, the increasing of the deformation $d_{i}^{y}$ is faster than that of the $d_{i}^{x}$ component. And for the ends of the cartilage ring, the greater force causes the larger deflection when pressing, and we take the 6th cartilage ring for the example as shown in Fig. $7 \mathrm{c}$. 


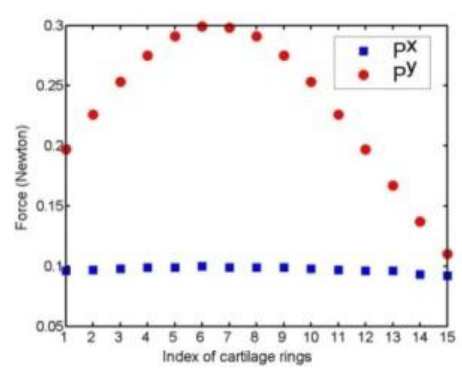

(a) Joint force rendering

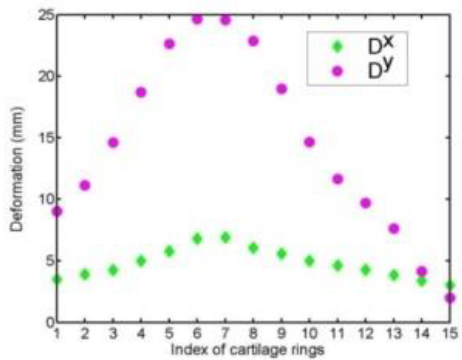

(b) Joint deformation rendering

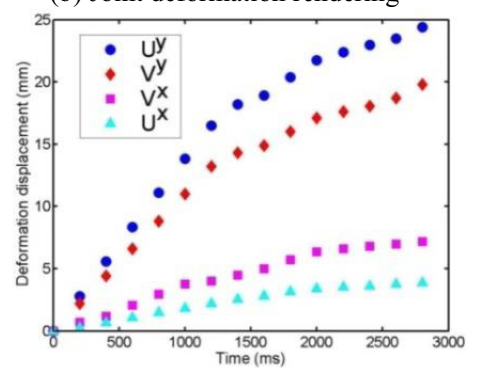

(c) Comparison of the deformation in two ends of the sixth cartilage ring

Figure 7. Force/deformation rendering

Table 1. Our experimental parameters

\begin{tabular}{|c|l|l|}
\hline \multicolumn{3}{|c|}{ Our experimental parameters } \\
\hline Symbols & \multicolumn{1}{|c|}{ Parameters } & Related value \\
\hline$M_{0}$ & Trachea mass & $30 \mathrm{~g}$ \\
\hline$L_{0}$ & Trachea length & $160 \mathrm{~mm}$ \\
\hline$D_{0}$ & Inner diameter of the trachea & $20 \mathrm{~mm}$ \\
\hline$h_{C}$ & Thickness of the cartilage rings & $2.6 \mathrm{~mm}$ \\
\hline$h_{M}$ & Thickness of muscular layer & $2 \mathrm{~mm}$ \\
\hline$A_{C}$ & Area of the cartilage rings & $4 \mathrm{~mm}^{2}$ \\
\hline$A_{L}$ & Area of the cartilage ligament & $6 \mathrm{~mm}^{2}$ \\
\hline$E_{C}$ & Young Module of cartilage rings & $5.8 \mathrm{MPa}$ \\
\hline$E_{L}$ & Young Module of ligament & $0.65 \mathrm{MPa}$ \\
\hline$\beta$ & Friction parameter & 0.66 \\
\hline
\end{tabular}

In all experiments, we use the real-time GJK method [10] to realize the contact detection and provide the distance information between the two objects, and then compute the penetration depth by Duffing Equations. The computation about skeleton model has an update rate of approximately $10 \mathrm{kHz}$. However, it is negligible compared with the time needed for real -time contact detection and shape updating for the trachea and rigid tool models in the order of $\mathrm{kHz}$, where the number of triangles in the trachea mesh model is 6856. Table 1 lists the parameters used in our experiments.

\section{Conclusions}

Trachea is a non-homogenous tube form larynx to the bronchial tubes, which consists of a supporting layer of connective and ligament tissue in which elastic cartilage rings are embedded and encircle the front of the tube. In this paper, we presented a novel skeleton model for a real-time virtual trachea surgical simulation. Our focus is to establish the trachea model, and use the skeleton approach to simulate the trachea deformation. The approach is able to model one contact applied by the virtual rigid finger, which can be handled by a user via a haptic device, and achieve the global or local shape deformation of the trachea model due to contacts. In the virtual surgery system, we implemented the approach and demonstrated its effectiveness, it is possible to perform surgical maneuvers at a speed approaching that of the actual procedure, while experiencing a sense of touch.

Future research will focus on implementing an implicit integration scheme for the simulation of visco-elastic materials. And considering the incision to keep the surgery integrity.

\section{Acknowledgment}

The research work was supported by National Natural Science Foundation of China under Grant No. 61402426 and partially supported by Collaborative Innovation Center of Novel Software Technology and Industrialization.

\section{References}

1. J. Wu, C. Dick, R. Westermann, Efficient collision detection for composite finite element simulation of cuts in deformable bodies, Visual Computer, 29(6), pp. 739-749 (2013)

2. L.P. Nedel and D. Thalmann, Real time muscle deformations using mass-spring systems, Computer Graphics International, pp. 156-166 (1998)

3. J. Russo, R. Robinson and M. J. Oldham, Effects of cartilage rings on airflow and particle deposition in the trachea and main bronchi, Medical Engineering and Physics, 30(5), pp. 581-589 (2008)

4. Q. Luo and J. Xiao, Contact and Deformation Modeling for Interactive Environments, IEEE Transactions on Robotics, 23, 3, pp.416-430 (2007)

5. Z.R. Liu, Z.Z. Teng, Y.Q. Wang and W.C. Tang, The circumferential residual strain and its nonuniform distribution in rat trachea, Chinese Journal of Biomedical Engineering, 22, 2, pp. 149152 (2003)

6. Y.C. Fung, Biomechanics, pp.400-406 (1981)

7. L.C. Teague, Jr., C.W. Pidgeon, Structured analysis methods for computer information systems , 1985 
8. A. E. Jr. Anuta, Compatibility of the modal equations, Computers and Structures, 33, 5, pp. 1229-1232 (1989)

9. A.Watt, 3D Computer Graphic, 3rd ed. Reading, MA: Addison Wesley, 2000
10. S. Cameron, Enhancing GJK: Computing minimum and penetration distances between convex polyhedra, Proc. IEEE International Conference on Robotics and Automation, pp. 3112-3117 (1997) 